\title{
¿Cuánto importa la competencia digital docente? Análisis de los programas de formación inicial docente en Uruguay
}

\author{
How does the digital teaching competence matter? An analysis \\ of initial teacher training programs in Uruguay
}

\author{
(iD) $M^{a}$ Julia Morales \\ Departamento de Sociología, Universidad de la República, Uruguay \\ mariajulia.morales@cienciassociales.edu.uy \\ Ana Rivoir \\ Departamento de Sociología, Universidad de la República, Uruguay \\ ana.rivoir@cienciassociales.edu.uy \\ (D) José Luis Lázaro-Cantabrana \\ Departamento de Pedagogía, Universidad Rovira i Virgili, España \\ joseluis.lazaro@urv.cat \\ (iD) Mercè Gisbert-Cervera \\ Departamento de Pedagogía, Universidad Rovira i Virgili, España \\ merce.gisbert@urv.cat
}

\section{RESUMEN}

El presente artículo presenta el análisis de la inclusión de las tecnologías de la información y la comunicación (TIC) en los programas de formación inicial docente (FID) en Uruguay. El objetivo es cómo la competencia digital docente (CDD) se incorpora en los programas de los cursos como parte de la formación de estudiantes, en el contexto de implementación de una política educativa pública de inclusión digital en el país. A través del análisis de contenido y bajo la metodología propuesta por Krippendorff (1990), se analizan los programas utilizando una matriz de indicadores realizada para medir el nivel de CDD. Las dimensiones consideradas son los fundamentos, objetivos y los contenidos. Del análisis se pudo inferir inconsistencias en la cobertura de la CDD entre los componentes de los programas analizados. Estas se presentan tanto a nivel de dimensiones como de indicadores, especialmente en los programas de las materias "Informática” y "Educación y tecnologías", no así en el programa de "Educación e integración de tecnologías digitales". En el programa de "Informática" se puede apreciar que los componentes analizados presentan escasa relación con la CDD, de acuerdo a la matriz utilizada para el análisis. Como conclusión más relevante destacamos que los programas analizados, si bien de forma dispar incluyen la mayoría de los componentes de la CDD, no responden a las necesidades formativas actuales en lo que a formación por competencias se refiere.

PALABRAS CLAVE competencia digital, universidad, educación superior, alfabetización digital. 


\begin{abstract}
This article presents the analysis of the inclusion of information and communication technologies (ICT) in the initial teacher training programs (FID) in Uruguay. The objective is to know how the digital teaching competence (CDD) are incorporated into the programs of the courses as part of the training of students, in the context of implementing a public education policy of digital inclusion in the country. Through the content analysis and under the methodology proposed by Krippendorff (1990), we analyse the programmes using a matrix of indicators to measure the CDD level. The dimensions considered are the foundations, objectives and contents. Based on the analysis, it was possible to infer inconsistencies in the coverage of the CDD among the components of the analysed programs. These are presented both at the level of dimensions and indicators, especially in the programs of the subjects "Information Technology", Education, and Technology, but not in the "Education and Integration of Digital Technologies" program. In the "Computing" program, the components have some relation with the CDD, according to the matrix used for the analysis. As a most relevant conclusion, we emphasize that the programs, although in different ways, include most of the components of the CDD but do not respond to the current training needs in terms of competency-based training.
\end{abstract}

KEYWORDS digital competence, university, higher education, digital literacy.

\title{
1. INTRODUCCIÓN
}

Uruguay es un referente en la región por sus políticas en informática educativa. El país ha experimentado un extenso desarrollo informacional en la última década, evidenciado en distintos indicadores nacionales e internacionales. Esto se sustenta en la expansión vía mecanismos del mercado y de políticas públicas. Varios estudios constatan la acelerada expansión de las tecnologías digitales y las mejoras en la infraestructura (Rivoir, 2013, 2017; Rivoir et al., 2017).

Desde 2007 se implementa en Uruguay el Plan Ceibal, mediante el cual se provee de un computador portátil a estudiantes del sistema escolar, docentes y estudiantes de formación docente. Se universaliza la conectividad de los centros educativos, aumentan los contenidos digitales educativos disponibles y se brinda formación y programas específicos en distintas áreas. Las investigaciones vinculadas a estas políticas (Morales, 2017; Rivoir, 2015; Rivoir y Lamschtein, 2014; Uriarte y Morales 2014), son concluyentes respecto de que no es suficiente con la mera incorporación de las tecnologías para favorecer los procesos de aprendizaje y destacan la importancia del contexto institucional del centro educativo y del hogar, el involucramiento de los docentes, los métodos de enseñanza y la formación docente, entre otros.

Formación docente fue el último nivel en incorporarse a esta política en 2010. En los planes de estudio de FID en Uruguay, solo se prevé programas específicos durante dos años en los institutos normales (II. NN.), un año en los institutos de formación de profesorado, un año en el instituto de maestro técnico y un año en el instituto de formación de educador social, con lo cual se dificulta la formación y apropiación de las TIC. Esta realidad evidencia las carencias en los contenidos, así como en la forma de inclusión de las TIC en los procesos de enseñanza aprendizaje en FID. Indagar cómo incluyen la competencia digital en la FID en Uruguay, a través del análisis de los programas, permitirá profundizar en el conocimiento sobre el alcance de dicha formación y, por tanto, sus posibilidades de incidencia en las transformaciones necesarias en un contexto digital. 


\section{MARCO TEÓRICO}

El acceso a los dispositivos móviles tecnológicos digitales se ha expandido y continúa haciéndolo en forma acelerada (ITU, 2017). Algunos estudios ven en este fenómeno una oportunidad para la enseñanza y la formación, tomando en consideración su expansión entre la población tanto como su ubicuidad (Horizon Report, 2016; Telefónica, 2016; Unesco, 2015). Esta universalización del acceso por parte de los estudiantes y docentes (Lázaro Cantabrana et al., 2015) interpela a las políticas pues obliga a repensarlas en función de este nuevo contexto tecnológico (Pedró, 2012).

Sostiene Selwyn (2013) que hay un consenso relativo en cuanto al rol de las tecnologías digitales en el aprendizaje a lo largo de toda la vida, desde la escuela y universidades hasta su rol en la formación permanente por Internet. Esto plantea nuevos desafíos a los sistemas educativos (Gisbert y Bullen 2015). Se requiere de infraestructura y condiciones de conectividad, pero también de recursos didácticos y de formación del profesorado, tanto como de la adquisición de competencias digitales (INTEF, 2016). Los cambios curriculares y en la formación del profesorado a los efectos de mejorar sus habilidades y capacidad de actuación crítica en el entorno digital constituyen factores centrales (Maris Briones et al., 2008). El liderazgo docente en este proceso es muy importante y, para ello, deben estar formados y ser capaces de análisis crítico sobre el potencial de las tecnologías digitales para la enseñanza y el aprendizaje (Lugo y Ruiz, 2016).

A tales efectos, debe cambiar la metodología docente y se requiere formación y seguimiento para la aplicación de los nuevos recursos y estrategias (Sonsoles Guerra et al., 2010).

\subsection{Las competencias digitales en la formación}

Resulta clave avanzar en torno a la discusión teórica de las categorías de análisis de procesos de incorporación de las tecnologías digitales. En particular las relacionadas a la adquisición de habilidades y competencias digitales y la apropiación de tecnologías digitales en educación, así como profundizar en los distintos métodos y técnicas para su análisis (Cabero-Almenara y Palacios-Rodríguez 2020; Gisbert y Esteve, 2011; Gisbert et al., 2016; Gisbert Cervera y Lázaro Cantabrana, 2015; Lázaro-Cantabrana et al., 2019; Rivoir, 2015, 2017; Silva et al., 2016).

El concepto de competencia digital ayuda a precisar algunas de las transformaciones en curso, así como aquellas que son necesarias en un contexto progresivamente digitalizado. Para Fraser et al. (2013) constituyen una competencia básica de los ciudadanos del siglo XXI.

Ferrari (2012) define las competencias digitales como el conjunto de conocimientos, habilidades y actitudes necesarias, para que la persona sea funcional en un entorno digital y Van Dijk (2008), indica que son centrales, a ser tomadas en cuenta para la reducción de la desigualdad digital en las sociedades contemporáneas.

Este autor refiere a cinco tipos de competencias digitales y sostiene son adquiridas progresivamente:

1. Las operacionales y formales que son las más básicas y están asociadas a los aspectos técnicos más elementales y las acciones sobre el hardware y con los programas.

2. Son las competencias informativas, que tienen que ver con la capacidad de búsqueda, selección y procesamiento de la información en Internet. 
3. Las competencias comunicacionales están relacionadas al envío, el contacto, la creación de identidades en línea y opinar en Internet.

4. Las competencias estratégicas constituidas por la capacidad para usar el medio digital con el fin de lograr ciertos objetivos y para obtener resultados en la posición social u obtener un rédito social específico.

5. Competencias para la creación de contenidos, conformadas por la capacidad de hacer contribuciones a Internet basándose en una planificación o diseño en particular (Van Dijk, 2008).

Es así que la competencia digital implica aspectos tecnológicos, informacionales multimediales y comunicativos lo que conlleva un proceso complejo de alfabetización múltiple (Gisbert y Esteve, 2011; Lázaro, 2015).

Varios autores (Llorente, 2008; Salinas y Silva, 2014) sostienen que, al implicar la preparación de las personas para las sociedades contemporáneas, involucra directamente a la formación inicial docente. También Suárez et al. (2010) afirman que es clave formar docentes con conocimientos y habilidades para favorecer el uso de las tecnologías digitales en los procesos de enseñanza aprendizaje para poder incorporarlas como un recurso innovador que propicie el desarrollo de competencias digitales por parte de los estudiantes. Por su parte, Gisbert et al. (2016) afirman que los docentes deben trascender la alfabetización digital básica e integrar a sus prácticas en clase, a su didáctica, las tecnologías digitales.

Por tanto, desarrollar la competencia digital docente (CDD) resulta un paso ineludible. Los profesionales de la educación necesitan adquirir las habilidades, actitudes y conocimientos que les permita promover un ambiente de aprendizaje en un contexto enriquecido por las tecnologías digitales. El uso de las tecnologías digitales debe ser aprovechado para la mejora y transformación de sus prácticas docentes, su desarrollo profesional y su identidad docente (Silva et al., 2017).

\subsection{Antecedentes}

En el contexto uruguayo, se realizaron dos estudios en relación a la integración de las TIC en los procesos de enseñanza aprendizaje y, específicamente, sobre el desarrollo de la CDD en FID.

Cabrera et al. (2018) en su análisis del lugar asignado a las tecnologías digitales en los planes y programas de formación inicial docente (FID) de profesorado de educación media en Uruguay encuentran que, en el programa de "Informática", se presenta de forma instrumental la adquisición de competencias digitales. Sostienen que en su redacción dejan entrever una necesaria relación con el resto de las asignaturas, lo hace de forma generalizada y no se visualiza la misma o una coordinación entre los diferentes programas.

El otro antecedente específico es el "Estudio comparado de la competencia digital docente en formación inicial en el contexto Uruguayo y Chileno" (Silva et al., 2018), donde se construye la matriz de indicadores que es la base del estudio aquí presentado (tabla 1). 
TABLA 1. Matriz de indicadores

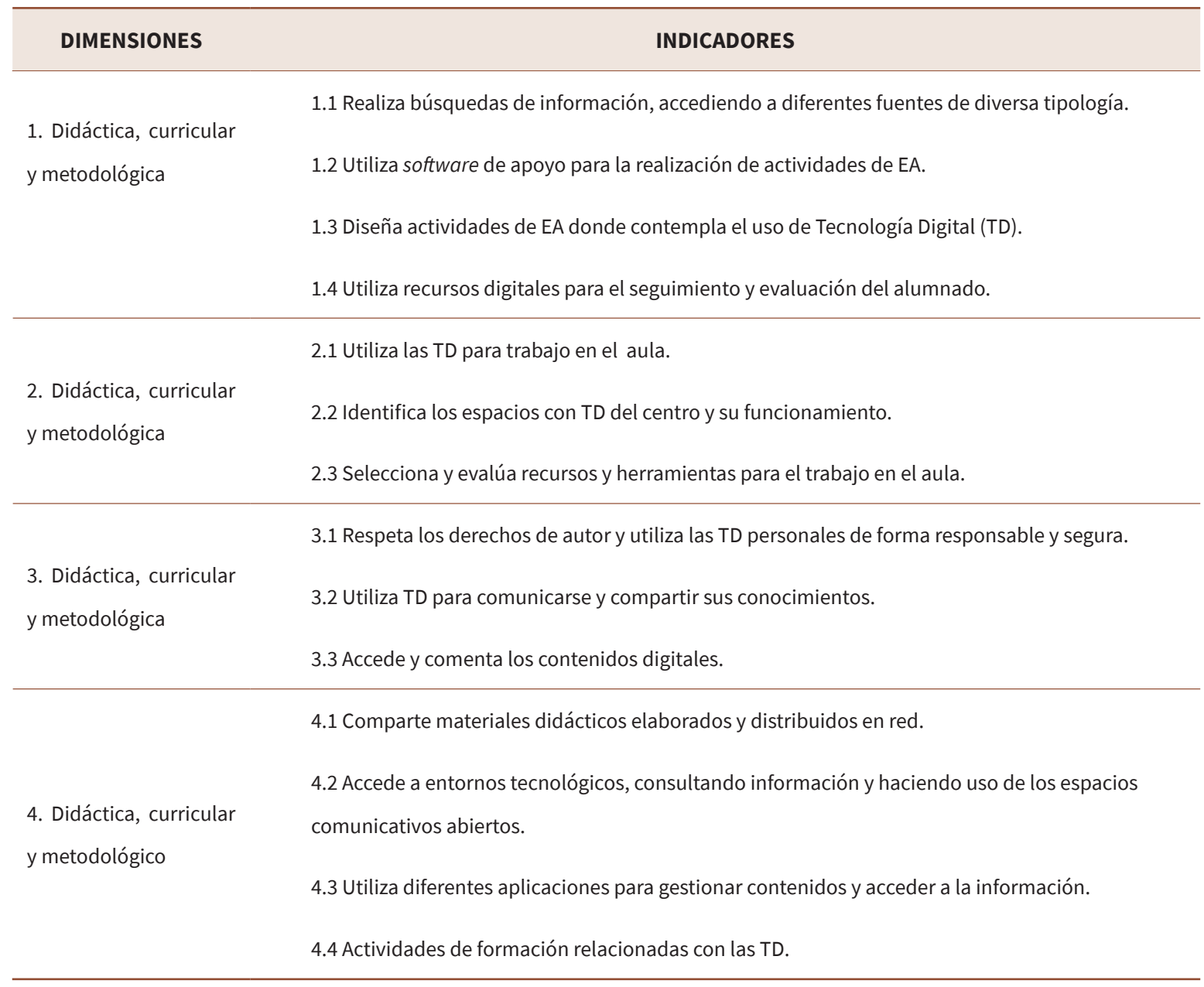

Silva et al (2016), en base a una revisión panorámica y a un análisis crítico documental, obtuvieron lineamientos para diseñar una matriz de indicadores de la competencia digital docente (CDD) en la formación inicial docente (FID). Para ello se tomaron en cuenta documentos oficiales de ministerios de educación y organizaciones vinculadas a las tecnologías digitales y su relación con la educación y orientaciones de instituciones de formación docente universitarias, formulados como estándares. En base a ello, se encontraron 5 aspectos centrales en relación a la CDD: a) pedagógica; b) desarrollo profesional; c) ética y seguridad; d) búsqueda y manejo de información y e) creación y comunicación de contenidos. A su vez se observaron dos maneras de abordarlos, los primeros centrados en los aspectos tecnológicos y los otros en aquellos pedagógicos en relación a la integración de las TIC en FID (Lázaro-Cantabrana et al., 2018; Silva et al., 2016).

\subsection{Objetivos}

Los resultados de investigación que se presentan en este artículo refieren al objetivo: análisis del modelo de formación que se aplica en Uruguay a los estudiantes de formación inicial docente en relación a la CDD. 


\section{MATERIAL Y MÉTODO}

El diseño metodológico se sostiene en una investigación que se vale de técnicas de recogida de datos cuantitativas y cualitativas. Si bien en el presente artículos nos referiremos al análisis de contenido en los programas de formación en FID y cómo se presenta la CDD en los mismos.

Se estructura en dos etapas: a) Etapa descriptiva en la que se propone evaluar el nivel de desempeño en relación a la CDD de los estudiantes de FID y describir el/los perfil/es de los docentes del Consejo de Formación en Educación en relación a sus CDD y busca entender la inserción de la CDD en los programas y/o planes de formación en educación y docentes en su forma y sentido y; b) Etapa propositiva en la que se propondrá un instrumento de intervención que tenga como objetivo, facilitar la nivelación al ingreso de los estudiantes de FID y sus docentes en CFE, en relación a la CDD.

Para comprender cómo es contemplada la CDD en los programas de formación en educación en FID en Uruguay, se estudiaron los siguientes programas relacionados con la inserción en TIC: a) Educación e integración de tecnologías digitales; b) Informática y c) Educación y tecnologías (tabla 2).

TABLA 2. Programas de TIC en FID según instituto y nivel de carrera

\begin{tabular}{lll}
\hline PROGRAMA & INSTITUCIÓN & AÑo QUE APLICA \\
\hline \multirow{2}{*}{ Informática } & & $2^{\circ}$ Magisterio \\
& IINN/ INET/ IFD/ CERP & $3^{\circ}$ Maestro técnico \\
\hline Educación e integración de tecnologías digitales & IINN & $3^{\circ}$ Profesorado \\
\hline Educación y tecnologías & IFES & $3^{\circ}$ Magisterio \\
\hline
\end{tabular}

La formación docente en Uruguay se encuentra bajo la órbita del Consejo de Formación en Educación (instituto de formación terciaria no universitaria).

Involucra seis tipos de instituciones: a) los institutos normales (II. NN.), con asentamiento en Montevideo (la capital del país), que forman maestros para la enseñanza primaria; b) el Instituto de Profesores Artigas (IPA), también en Montevideo, donde se forman los profesores para enseñanza secundaria; c) los centros regionales de profesores (CERP), que se encuentran en algunas ciudades del país (exceptuando la capital), distribuidos de forma estratégica de acuerdo a densidad poblacional y demandas y forman profesores para la enseñanza secundaria; d) los institutos de formación docente (IFD), distribuidos en todo el país (exceptuando la capital), que forman maestros para enseñanza primaria y, de acuerdo a algunos territorios, en el ciclo inicial pueden albergar materias comunes para profesorado; e) el Instituto de Formación en Educación Social (IFES), de donde egresan los educadores sociales y f) el Instituto Normal de Enseñanza Técnica (INET), de donde egresan los maestros técnicos que ejercen en su gran mayoría enseñanza en la Universidad del Trabajo del Uruguay que, a pesar de su nombre, es equivalente a educación media. Asimismo, existen dos modalidades de cursado en FID: la presencial y la semi-presencial (gráfico 1). 
GRÁFICO 1. Evolución de la matrícula del Consejo de Formación en Educación por Institución 2000-2018

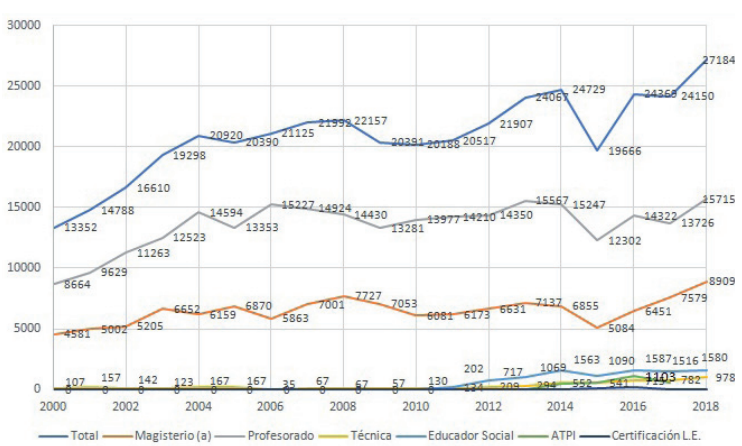

FUENTE: División Estudiantil del CFE hasta 2016. Elaboración departamento Información y estadística CFE a partir de 2017 (Lamas y Evans, p. 10)

(a) Magisterio incluye las carreras de Maestro de Educación Primaria y Maestro de Primera Infancia, especialidad que se ofrece a partir de 2017. (b) Educación Técnica comprende las carreras de Maestro y Profesor Técnico y la Tecnicatura "ALTD".

\subsection{Instrumentos de recogida de datos}

Para el análisis de los programas y/o planes de FID de CFE se tuvo en cuenta la metodología propuesta por Krippendorff (1990), para el que esta técnica permite formular "a partir de ciertos datos, inferencias reproducibles y válidas que puedan aplicarse a su contexto" (p. 28). Establece que dichos datos "obliga a un receptor a realizar inferencias específicas en relación a su medio empírico" (p. 31) denominando a este medio empírico como el contexto de los datos.

Se aplicó, para realizar el análisis, las unidades específicas de muestreo, registro y contexto. Las unidades de muestreo, independientes una de otras y que son compartidas por los programas analizados son: a) Fundamentos o propósitos; b) Objetivos o resultados de aprendizaje y c) Contenidos. Una cuarta unidad de muestreo, que se dejó de lado al realizar el análisis, fue la de d) Metodología, debido a que solo 2 de los 3 programas la contenían (Miranda et al., 2018; Morales y Rivoir, 2018).

Dentro de cada unidad de muestreo, para el análisis, se consideran las llamadas unidades de registro y corresponden a segmentos específicos de contenido; a estas unidades de registro le corresponden, a su vez, una unidad de contexto que representa su contenido simbólico y le es característico (Miranda et al., 2018; Morales et al., 2018; Morales y Rivoir, 2018). En este estudio se consideraron 128 unidades de registro en las 3 unidades de muestreo analizadas.

Para el presente estudio se trabajó con unidades referenciales, uno de los cinco tipos establecidos por Krippendorff (1990) para identificar las unidades de contexto (físicas, sintácticas, proposicionales, temáticas y referenciales) y que corresponden a "expresiones que refieren a determinados objetos, sucesos, personas, ideas..." (p. 27).

Para la realización del análisis se elaboró una matriz para cada unidad de muestreo por cada programa analizado, que permitía relacionar a cada unidad de registro con el constructo teórico plasmado en la matriz de indicadores realizada en el "Estudio comparado de la competencia digital docente en formación en Chile y Uruguay" (Morales et al, 2018).

\section{RESULTADOS}

Para observar el nivel de CD en los programas de FID en CFE, en base a tres componentes, a) Objetivos; b) Fundamentos y, c) Contenidos; se analizaron 128 unidades de registro, de las cuales un $21 \%$ no corresponden con ninguno de los indicadores de la matriz de análisis, en ninguno de los componentes.

En dicho análisis se puede observar que, para las diferentes dimensiones, la presencia de unidades de registro en relación al componente contenidos es la de mayor presencia, a excepción de la dimensión 2, Planificación, organización y gestión de espacios y recursos tecnológicos digitales, donde el componente Fundamentos, lo supera. 
GRÁFICO 2. Cobertura de la CDD (dimensiones) según componentes de las asignaturas de TIC en FID en Uruguay

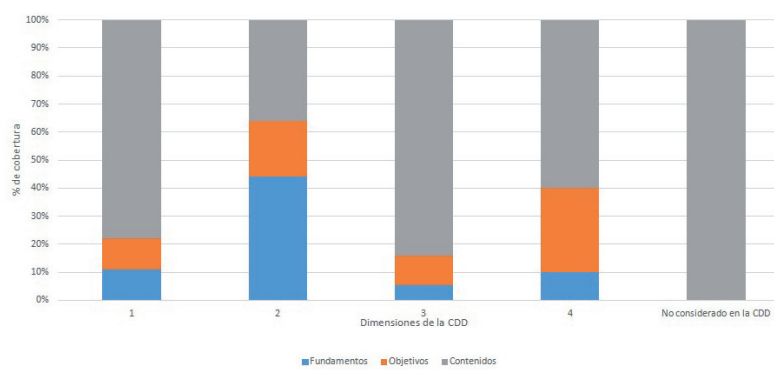

En el resto de las dimensiones de análisis el componente Fundamentos tiene menor presencia, seguido por el componente Objetivos que, como puede apreciarse en la gráfica, supera ampliamente en la dimensión 4, Desarrollo personal y profesional.

El análisis también permitió observar el nivel de $C D$ al interior de cada una de las dimensiones, es decir, en relación con cada uno de los indicadores mencionados en la tabla 1.

Se puede apreciar que el componente de contenidos se encuentra presente en todas las dimensiones de análisis en forma mayoritaria. Sin embargo, el componente Objetivos se presenta de forma dispar en los diferentes indicadores, con mayor presencia en la Dimensión 3 y 4 y que el componente Fundamentos tiene mayor presencia en la Dimensión 2.

Al profundizar y observar en los distintos indicadores la presencia de los diferentes componentes, podemos apreciar que en la Dimensión 1, Didáctica, curricular y metodológica, los componentes de objetivos y fundamentos solo están presentes en el indicador 1.3, Diseña actividades de EA donde contempla el uso de Tecnología Digital (TD) (gráfico 3). Mientras que en las restantes dimensiones, si bien de forma desigual, se presentan en casi la totalidad de los indicadores los 3 distintos componentes (gráficos 4,5 y 6 ).

GRÁFICO 3. Cobertura de la CDD (dimensiones) según componente para la Dimensión 1

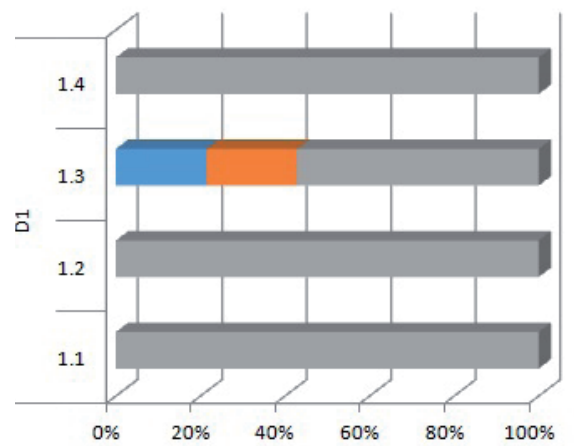

GRÁFICO 5. Cobertura de la CDD (dimensiones) según componente para la Dimensión 3

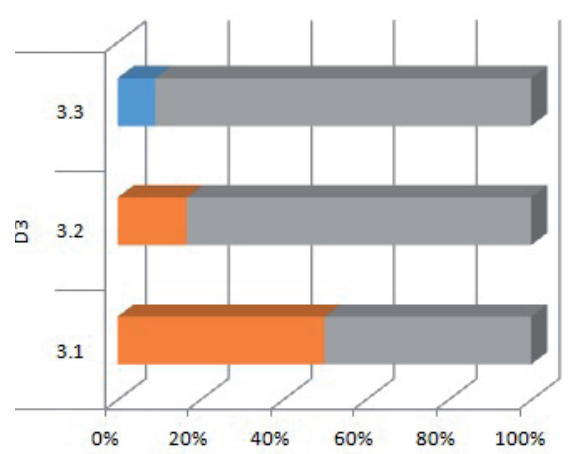

GRÁFICO 4. Cobertura de la CDD (dimensiones) según componente para la Dimensión 2

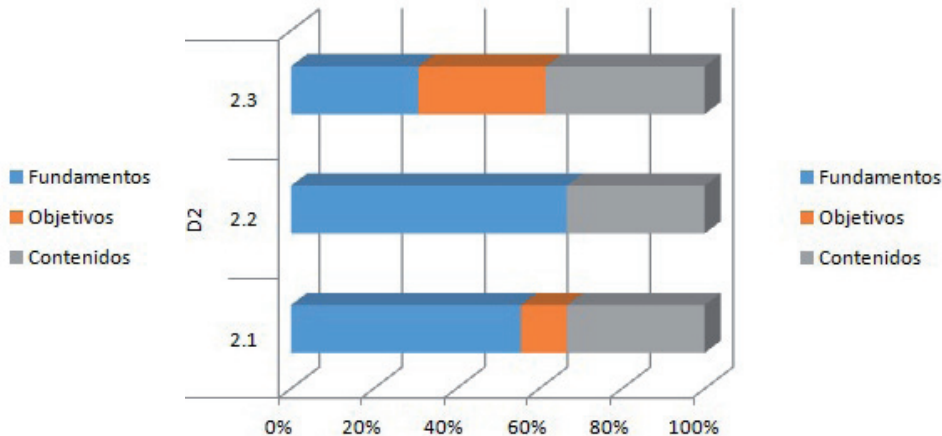

GRÁFICO 6. Cobertura de la CDD (dimensiones) según componente para la Dimensión 4

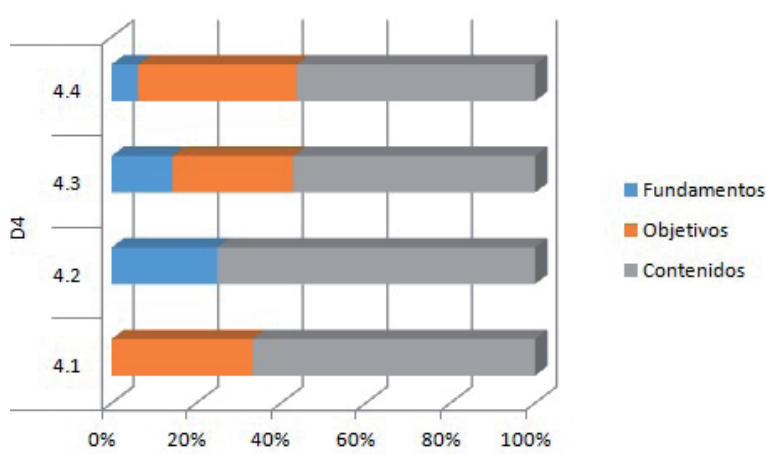


Aun así, en los indicadores 2.2, Identifica los espacios con TD del centro y su funcionamiento; 3.3 Accede y comenta los contenidos digitales y 4.2 Accede a entornos tecnológicos, consultando información y haciendo uso de los espacios comunicativos abiertos, solo están presentes los componentes Fundamentos y Contenidos.

Es así como en líneas generales podemos encontrar inconsistencias en la cobertura de la CD para los diferentes componentes, tanto a nivel de dimensión como de indicadores; presentándose la Dimensión 4, Desarrollo personal y profesional, como la más cubierta y la Dimensión 1, Didáctica, curricular y metodológica, como la menos cubierta en referencia a un equilibrio entre los 3 componentes estudiados.

GRÁFICO 7. Cobertura de la CDD (dimensiones) según componente para el Programa de Informática

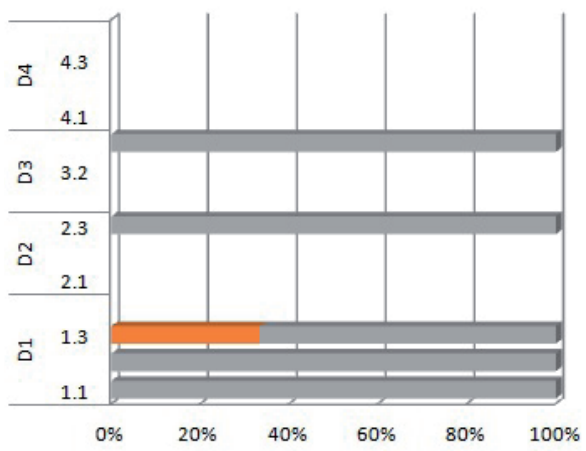

GRÁFICO 8. Cobertura de la CDD (dimensiones) según componente para el Programa de Educación y tecnología

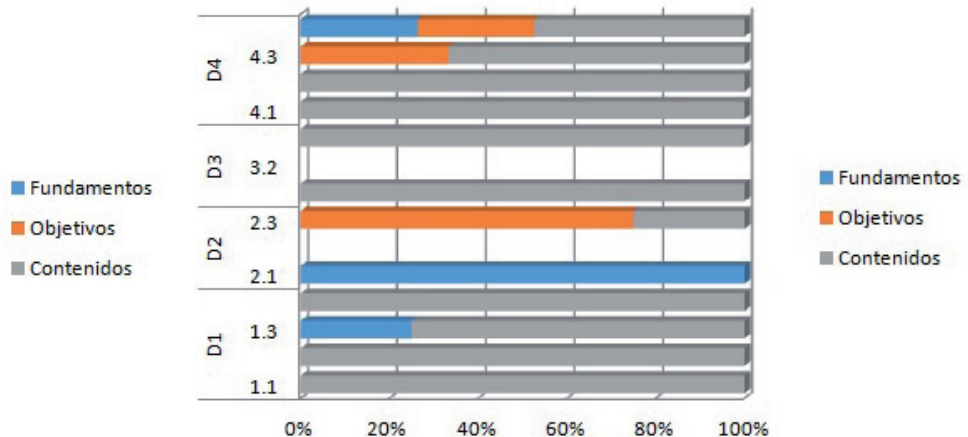

En el programa de "Informática" se puede apreciar que los componentes presentan escasa relación con las CDD de acuerdo a la matriz utilizada para el análisis. En este programa solo 5 de los 14 indicadores se encuentran presentes en el componente Contenidos, y uno solo de los indicadores presente en el componente Objetivos (gráfico 7).

En el programa "Educación y tecnologías" se puede inferir del análisis que, si bien están más presentes la mayoría de los indicadores en el componente contenidos, no es así para los restantes indicadores, ya que solo se presentan en los indicadores 1.3, Diseña actividades de EA donde contempla el uso de Tecnología Digital (TD); 2.1, Utiliza las TD para trabajo en el aula; 2.3, Selecciona y evalúa recursos y herramientas para el trabajo en el aula; 4.3, Utiliza diferentes aplicaciones para gestionar contenidos y acceder a la información

GRÁFICO 9. Cobertura de la CDD (dimensiones) según componente para el Programa de Educación e integración de tecnologías digitales

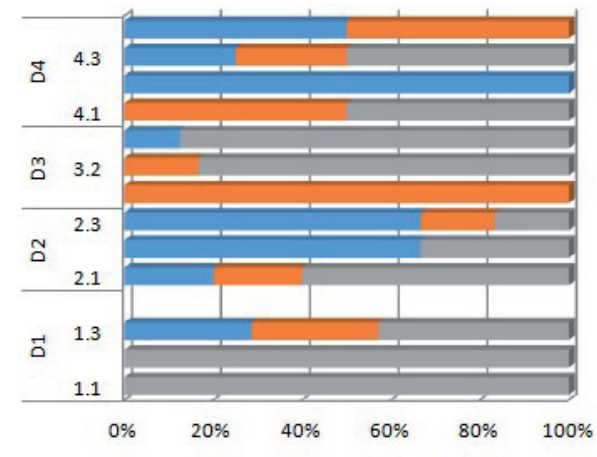

y 4.4, Realiza actividades de formación relacionadas con las TD. Y que la dimensión 3, Aspectos éticos, legales y seguridad, no está contemplada en ninguno de los componentes (gráfico 8).

En el programa "Educación e integración de tecnologías digitales", los indicadores de CDD se encuentran presentes más homogéneamente en casi la totalidad de los indicadores. El único indicador que no se encuentra en ninguno de los componentes analizados es el 1.4, Utiliza recursos digitales para el seguimiento y evaluación del alumnado (gráfico 9). 
En general, podemos afirmar que, a partir del análisis de la unidad de contexto a través de las unidades sintácticas del programa de "Informática", se evidencia el predominio de un paradigma alejado de una concepción de enseñanza por competencias. Mientras que el programa de "Educación y tecnologías” y el de “Educación e integración de tecnologías digitales", aunque con diferencias relevantes entre sí, están más próximos a un diseño que busca el desarrollo de la competencia digital, aunque no esté explícitamente indicado.

En ninguno de los programas se explicitan las competencias a lograr. Sin embargo, las mismas se infieren en dos de los programas a través del análisis del material (programas) y específicamente de las unidades de contexto y unidades sintácticas.

\section{DISCUSIÓN}

De forma general, los hallazgos confirman que los programas se alejan de un paradigma de enseñanza por competencias, desarrollo que es ineludible en la FID en la actualidad. Y si adherimos a su concepción como el conjunto de habilidades, actitudes y conocimientos que promueven un ambiente de aprendizaje, en un contexto enriquecido por tecnologías digitales, favoreciendo la mejora y transformación de sus prácticas docentes, el desarrollo profesional e identidad docente (Silva et al., 2016) debería, a través del análisis de los programas, inferirse contenidos que favorezcan el desarrollo de dichas habilidades, actitudes o conocimientos que promuevan por ejemplo la resolución de problemas en contextos profesionales o la vinculación teoría y práctica en el proceso de enseñanza - aprendizaje, etc.

En este sentido, uno de los hallazgos refiere a que los programas analizados no mencionan explícitamente la CDD o el desarrollo de la misma, aunque a nivel de unidades de contexto y sintácticas puede presentarse el desarrollo de la CDD. En alguno de los programas esto concuerda con los hallazgos del estudio de Silva et al. (2017). Allí se indica que, si bien los programas no explicitan la competencia digital, en uno de ellos se infiere a través de las unidades de contexto y de las unidades sintácticas el desarrollo de dicha competencia. En el caso del programa de Informática, mencionan "la unidad de contexto podría ser facilitadora de la CDD en la Dimensión didáctica, curricular y metodológica, ya que el estudiante aprendería conocimientos que puede aplicar a la enseñanza en los distintos campos de conocimiento realizando búsquedas de información a través de diferentes fuentes y/o utilizando recursos digitales para el seguimiento y evaluación" (p. 14).

Asimismo, en el estudio de Cabrera et al. (2018) se menciona que el programa de la asignatura Informática presenta de forma instrumental la adquisición de competencias digitales y que, a pesar de que en su redacción deja entrever la necesaria relación con el resto de las asignaturas, lo hace de forma generalizada y no se visualiza la misma o una coordinación entre los diferentes planes; "Búsqueda de información en Internet (...) Aplicación a distintas disciplinas y áreas de conocimiento” (Cabrera et al., 2018, p. 2).

Otro de los hallazgos refiere a que en el programa Informática los componentes presentan escasa relación con la CDD de acuerdo a la matriz utilizada para el análisis. Se evidencia el carácter instrumental del mismo y el alejamiento de un paradigma de enseñanza por competencias, hallazgo que comparte el estudio de Cabrera et al. (2018) donde menciona "En otro orden, del análisis realizado es posible apreciar que el Plan 2008, vigente en la actualidad, no se enmarca en el paradigma de competencias y, por tanto, son escasas las menciones que pueden interpretarse como un aporte al desarrollo genuino de la CD” (p. 29). 
Hemos centrado esta discusión en el programa de Informática, ya que es el único que está presente en los tres estudios que hay en el país al respecto. Sin embargo, al estudiar los dos restantes programas, Educación y tecnología y Educación e integración de tecnologías digitales, el hallazgo general nos dice que existen inconsistencias a nivel de dimensión e indicadores para la cobertura de la CDD, de acuerdo a la matriz utilizada en el estudio. Esto evidencia que, si bien en las unidades de contexto, a través de las unidades sintácticas, se percibe el desarrollo de algunos conocimientos y habilidades aplicables en la práctica profesional del futuro docente, esta es escasa y en pocos indicadores.

\section{CONCLUSIONES}

En el análisis general la dimensión 1, Didáctica, curricular y metodológica, es la de comportamiento menos homogéneo, ya que solo el indicador 1.3, tiene cobertura a nivel de fundamentos, objetivos y contenidos, mientras que las dimensiones más cubiertas, parecen ser la dimensión 2, Planificación, organización y gestión de espacios y recursos tecnológicos digitales y 4, Desarrollo personal y profesional.

Asimismo, se aprecian inconsistencias en la cobertura de la CDD entre los componentes de los programas analizados. Estas se presentan tanto a nivel de dimensiones como de indicadores, especialmente en los programas de “Informática” y “Educación y tecnologías”. No así en el programa de "Educación e integración de tecnologías digitales".

Si bien en los distintos programas la cobertura de las dimensiones e indicadores de la CDD en los componentes de Fundamentos y Objetivos, esto se revierte en el componente de Contenidos.

Se concluye que los programas analizados no responden a las necesidades actuales de creación de CDD, de forma que los egresados cuenten con esas herramientas (Ferrari, 2012; Gisbert et al., 2016; Horizon Report, 2016; Maris Briones et al., 2008; Silva et al, 2016; Sonsoles Guerra et al., 2010; Telefónica, 2016; Unesco, 2012). Es así que aparece como necesaria una adecuación curricular de los programas de formación inicial docente en relación con la CDD. De acuerdo a los hallazgos, hasta el momento los programas muestran un alejamiento de la concepción de enseñanza por competencias.

En síntesis, los programas formativos no se basan en el desarrollo de competencias. Del análisis se desprende que la CDD no se explicita ni el desarrollo de la misma. Apenas se infiere en uno de ellos, "Educación e integración de tecnologías digitales" a través de sus unidades de contexto y sintácticas, desarrollando habilidades que a los estudiantes le aportaría conocimiento para sus prácticas docentes.

Por tanto, dada la necesidad en la sociedad actual de docentes competentes digitalmente (Gisbert et al., 2016; INTEF, 2016; Llorente, 2008; Lugo y Ruiz, 2016; Maris Briones et al., 2008; Salinas y Silva, 2014; Selwyn, 2013; Silva et al., 2016; Suárez et al., 2010) y en Uruguay particularmente dados los esfuerzos gubernamentales y de las autoridades de la educación en la implementación de políticas públicas para incluir digitalmente a la ciudadanía (Morales, 2017; Rivoir y Lamschtein, 2012, 2014; Silva et al., 2016; Uriarte y Morales, 2014), el mayor reto tanto a nivel administrativo (autoridades educativas) como a nivel académico (centros de formación inicial docente y de formación permanente del profesorado) que conlleva para la FID en Uruguay, a la luz de los hallazgos, es incluir en los programas de formación el desarrollo de la CDD, su evaluación y su eventual certificación. 


\section{REFERENCIAS}

Cabero-Almenara, J., y Palacios-Rodríguez, A. (2020). Marco Europeo de Competencia Digital Docente «DigCompEdu» y cuestionario «DigCompEdu Check-In». EDMETIC, Revista de Educación Mediática y TIC, 9(1), 213-234. https://doi. org/10.21071/edmetic.v9i1.12462

Cabrera, C., Cabrera, A., Carámbula, S., Pérez, A., y Pérez, M. (2018). Tecnologías digitales: Análisis de planes de profesorado en Uruguay. Cuadernos de investigación educative, 9(2), 13-32.

Ferrari, A. (2012). Digital Competence in Practice: An Analysis of Frameworks. JRC-IPTS.

Fraser, J., Atkins, L., y Richard, H. (2013). DigiLitleicester. Supporting teachers, promoting digital literacy, transforming learning. Leicester City Council.

Gisbert Cervera, M., y Lázaro Cantabrana, J. (2015). Professional development in teacher digital competence and improving school quality from the teachers' perspective: a case study. Journal of New Approaches in Educational Research, 4(2), 115-122. http://dx.doi.org/10.7821/naer.2015.7.123

Gisbert, M., Esteve, F., y Lázaro, J. (2016). La competencia digital de los futuros docentes: ¿cómo se ven los actuales estudiantes de educación? Perspectiva Educacional, 55(2), 34-52. http://dx.doi.org/10.4151/07189729-Vol.55-Iss.2-Art.412.

Gisbert, M., y Bullen, M. (Ed.) (2015). Teaching and learning in digital worlds. Strategies and issues in higher education. Publicaciones Universitat Rovira i Virgili.

Gisbert, M., y Esteve, F. (2011). Digital learners: la competencia digital de los estudiantes universitarios. La cuestión universitaria, 7, 48-59.

Horizon Report (2016). Horizon Report. Higher Education Edition. http://cdn.nmc.org/media/2016-nmc-horizon-report-HEES.pdf

INTEF (2016). Resumen Informe. Competencias para un mundo digital. Instituto Nacional de Tecnologías Educativas y Formación de Profesorado. http://blog.educalab.es/intef/wpcontent/uploads/sites/4/2016/10/2016_1003-Competencias_mundo_digital_OCDE_INTEF.pdf
ITU (2017). World Telecommunication Development Conference (WTDC-17): Final Report. Ginebra.

Krippendorff, K. (1990) Metodología de análisis de contenido: teoría y práctica. Paidós.

Lamas, C., y Evans, T. (2018). Informe de matrícula del CFE. Matrícula inicial y características sociodemográficas de los estudiantes. Consejo de Formación en Educación. Montevideo - Uruguay.

Lázaro Cantabrana, J. L., Estebanell Minguell, M. y Tedesco, J. C. (2015). Inclusión y cohesión social en una sociedad digital. RUSC. Universities and Knowledge Society Journal, 12(2), 4459. http://dx.doi.org/10.7238/rusc.v12i2.2459

Lázaro, J. (2015). La competència digital Docent com a eina per garantir la qualitat en I'ús de les TIC en un centre escolar [Tesis doctoral]. Universitat Rovira i Virgili.

Lázaro-Cantabrana, J., Gisbert-Cervera, M., y Silva-Quiroz, J. (2018). Una rúbrica para evaluar la competencia digital del profesor universitario en el contexto latinoamericano. Edutec. Revista Electrónica de Tecnología Educativa, 0(63), 1-14. http://dx.doi.org/10.21556/edutec.2018.63.1091

Lázaro-Cantabrana, J., Usart-Rodríguez, M., y Gisbert-Cervera, M. (2019). Assessing Teacher Digital Competence: the Construction of an Instrument for Measuring the Knowledge of Pre-Service Teachers. Journal of New Approaches in Educational Research, 8(1), 73-78. http://dx.doi.org/10.7821/ naer.2019.1.370

Llorente, M. C. (2008). Aspectos fundamentales de la formación del profesorado en TIC. Pixel-Bit: Revista de medios y educación, 31, 121-130.

Lugo, M., y Ruiz, V. (2016). Reflexiones en torno a los escenarios educativos de integración TIC. En Unesco/ Fundación Telefónica (Coord.), Experiencias Evaluativas de Tecnologías Digitales en la Educación (pp. 87-96). Fundação Telefônica Vivo.

Maris Briones, S., Martínez, M., Siñanes, G., y Rivero, M. (2008). Nuevos espacios de interactividad para la práctica pedagógica universitaria. Pixel-Bit. Revista de Medios y Educación, 33, $165-172$.

Miranda, P., Silva, J., y Contreras, S. (2018). La presencia de la competencia digital docente en los programas de formación 
inicial del profesorado en las universidades públicas chilenas. En X. Carrera, F. Martínez y J. L. Coiduras (Coords.), EDUcación con TECnología. Un compromiso social. Aproximaciones desde la investigación e innovación (pp. 421-430) Edicions de la Universitat de Lleida. https://doi.org/10.21001/edutec.2018

Morales, M. J. (2017). Apropiación social de la tecnología por parte de la población de la localidad de Cebollatí en Uruguay. Análisis empíricos y reflexiones teóricas (pp. 51-62). Ediciones Universitarias - UCUR.

Morales, M. J., y Rivoir, A. L. (2018). La competencia digital en formación inicial docente. ¿Los programas y planes de formación de estudiantes y docentes se ajustan a las nuevas formas de aprender y estudiar con TIC? En X. Carrera, F. Martínez y J. L. Coiduras (Coords.), EDUcación con TECnología. Un compromiso social. Aproximaciones desde la investigación e innovación (pp. 291-297) Edicions de la Universitat de Lleida. https://doi.org/10.21001/edutec.2018

Morales, M., Silva, J., Gisbert, M., Lázaro, J. L., Onetto, A., Rivoir, A., y Miranda, P. (2018). Estudio comparado de la competencia digital docente en formación en Chile y Uruguay. EDUcación y TECnología. Propuestas desde la investigación y la innovación educativa (pp. 139-141), Universidad de Santiago de Chile.

Pedró, F. (2012) Prólogo. En G. Sunkel, G y D. Trucco (Ed.), Las tecnologías digitales frente a los desafíos de una Educación Inclusiva en América Latina. CEPAL.

Rivoir, A. (2015). La desigualdad digital a la luz de las iniciativas para su reducción. En S. Lago y N. Correa (Coords.), Desafíos y dilemas de la Universidad y la Ciencia en América Latina y el Caribe en el siglo XXI. Teseo.

Rivoir, A. (2016). Revisión comparativa de iniciativas nacionales de aprendizaje móvil en América Latina. El Caso de la política TIC en Perú. Editorial: Paris: UNESCO

Rivoir, A. (2017). Reflexiones teóricas y metodológicas a partir de la investigación social sobre inclusión y desigualdad digital. En R. Cabello y A. López (Eds.), Contribuciones al estudio de procesos de apropiación de tecnologías (pp. 53-60). Gato Gris.

Rivoir, A. (Coord.) (2013) Plan Ceibal e Inclusión Social. Perspectivas interdisciplinarias. Plan Ceibal. Montevideo: Universidad de la República.
Rivoir, A. y Lamschtein (2012). Cinco años del Plan Ceibal. Algo más que una computadora. Montevideo: UNICEF

Rivoir, A., Escuder, S., y Rodríguez, F. (2017). Usos percepciones y valoraciones de Wikipedia por profesores universitarios. Innovación educativa, 17, 169-190.

Rivoir, A., y Lamschtein, S. (2014). Brecha Digital e inclusión social, contribuciones y dilemas de las políticas 1 a 1 . El caso de Ceibal en Uruguay. Razón y Palabra, 87.

Salinas, J., y Silva J. (2014). Innovación con TIC en la formación inicial docente en Iberoamérica. En J. Silva y J. Salinas (Coord.), Innovación con TIC en Formación Inicial Docentes: Aspectos teóricos y casos concretos (pp. 1233). Ministerio de Educación Chile.

Selwyn, N. (2013). Education in a Digital World: Global Perspectives on Technology and Education.Routledge.

Silva, J., Miranda, P., Gisbert, M., Morales, M., y Onetto, A. (2016). Indicadores para evaluar la competencia digital docente en la formación inicial en el contexto Chileno - Uruguayo. RELATEC: Revista Latinoamericana de Tecnología Educativa, 15(3), 55-67. http://dx.doi.org/10.17398/1695-288X.15.3.55

Sonsoles Guerra, S., González, N., García, R. (2010). Utilización de las TIC por el profesorado universitario como recurso didáctico. Comunicar, 35, 141-148.

Suárez, J. M., Almerich, G., Gargallo, B., y Aliaga, F. (2010). Las competencias en TIC del profesorado y su relación con el uso de los recursos tecnológicos. Archivos Analíticos de Políticas Educativas, 18(10), 1-33. https://doi.org/10.14507/epaa. v18n10.2010

Telefónica (2016). Informe de la sociedad de la información. http:// www.fundaciontelefonica.com/arte_cultura/publicacioneslistado/pagina-item-publicaciones/itempubli/558/

Unesco (2015). La Educación para Todos, 2000-2015: logrosydesafíos. http://unesdoc.unesco.org/images/0023/002324/232435s.pdf Uriarte P., y Morales, M. (2014). ¿Qué queremos decir con Integralidad? Una mirada a través del Proyecto Flor de Ceibo de la Universidad de la República. Revista Encuentros Uruguayos, $8(1), 54-78$.

Van Dijk, J. (2008). Digital divide in Europe. En A. Chadwick y Ph. Howard (Ed.), The handbook of Internet Policies. Routledge. 